\title{
Optimizing the Association Procedure for Low-Power 802.15.4 Nonbeacon Sensor Networks
}

\author{
Barbara Staehle \\ University of Würzburg, Institute of Computer Science, Würzburg Germany \\ bstaehle@informatik.uni-wuerzburg.de
}

\begin{abstract}
Building wireless sensor networks based on the IEEE 802.15.4 standard is an interesting option, as the standard enables low-power, low data rate wireless communication. Many authors analyzed and optimized the operational phase of such networks. In contrast, the initial phase, containing the 802.15 .4 association procedure, has mostly been neglected. In this paper, we therefore propose four optimization possibilities for the association procedure of a nonbeacon-enabled sensor network. Our results show that significant performance improvements in terms of association probability, speed, and energy consumptions can be achieved.
\end{abstract}

Keywords: Wireless Sensor Networks, Nonbeacon 802.15.4, Low-Power.

\section{Introduction}

The IEEE 802.15.4 Low-Rate Wireless Personal Area Networks standard specifies PHY and MAC layer protocols to enable wireless connectivity while guaranteeing "ease of installation, reliable data transfer, short-range operation, extremely low cost, and a reasonable battery life" [1]. Together with application layer protocols specified by ZigBee [2] and WirelessHART [3], it is seen as a promising option for wireless sensor networking and has become increasingly popular for industrial purposes, monitoring and control applications.

We recall shortly the most important 802.15.4 features: each Personal Area Network (PAN) has exactly one PAN coordinator which should be mains powered and may function as the data sink in a 802.15.4 Wireless Sensor Network (WSN). It is able to send out beacons, small command messages which are used for synchronization and organization purposes. In the beacon mode, beacons are regularly broadcasted in order to maintain a superframe structure allowing to meet low latency or bandwidth requirements. This overhead is avoided in the nonbeacon mode which is suitable for WSNs with less tight synchronization, bandwidth or delay requirements like environmental monitoring or scientific data collection applications 1]. As network topologies, the 802.15.4 standard allows either the star or the peer-to-peer topology. As the latter enables larger, more complex, robust and flexible network formations, it is explicitly proposed for WSNs. In this study, we focus on a WSN operating in the 802.15.4 nonbeacon mode where all sensor nodes are Full Functional Devices (FFDs), i.e. are able to

L. Fratta et al. (Eds.): NETWORKING 2009, LNCS 5550, pp. 626-638, 2009.

(C) IFIP International Federation for Information Processing 2009 
relay packets on behalf of other nodes. This setting offers the highest degree of flexibility and resilience.

In low-power WSN deployments, sensor nodes are battery powered or energy harvesting, saving energy is thus the main issue. In 802.15.4 power saving mechanisms are only existing for the beacon mode, for a nonbeacon-enabled network, no possibilities for energy savings are forseen [1]. Together with a sensor MAC protocol or a higher level sleep scheduling solution, nonbeacon-enabled 802.15.4 peer-to-peer networking is nevertheless an interesting option for low power-WSNs. Very few of the proposed 802.15.4 enhancements cover the initial stages of the network life cycle, but a 802.15.4 network can not become functional before the association procedure (reviewed in Section 3.1) has been completed successfully. This procedure is optimized for star topologies and is thus less effective in large nonbeacon-enabled topologies.

How a nonbeaconed 802.15.4 network can start up efficiently has never been considered in depth, this study therefore focuses on the startup phase of a nonbeacon-enabled low-power 802.15.4 WSN. Related work is reviewed in Section 2. The association procedure is described in Section 3. where we reveal issues and propose optimizations for large low-power networks. In Section 4 the methodology used for evaluating different optimization possibilities is introduced. Section [5] contains insights on the individual and combined effects of the considered parameters and demonstrates that any low-power 802.15.4 WSN can operate power efficiently from the beginning. We conclude our work and point out future research directions in Section 6 .

\section{Related Work}

Many performance evaluations of 802.15.4 have been published. As the 802.15.4 nonbeacon MAC protocol is barely more than plain CSMA-CA, most studies analyze and optimize aspects of the 802.15 .4 beacon mode. An exemplary study of the beacon mode is the work of Kohvakka et al. [4, who analyzed the performance of 802.15.4 for large-scale WSN applications. The authors established models for the device energy consumptions and goodput in beaconed cluster tree topologies. The results allow to choose two key 802.15.4 superframe parameters for increasing the network performance and energy efficiency.

An analysis of the nonbeacon-enabled channel access was presented by Latré et al. 5] who determined maximum throughput and minimum delay for a single connection. The authors showed that the small 802.15.4 packet sizes together with the large protocol overhead significantly decreases the bandwidth efficiency. The nonbeaconed channel access has also been studied by Kim et al. 6]. Using a Markov chain model, the authors proved that the number of devices in a star topology can be optimized in order to guarantee specific QoS conditions.

As the nonbeacon mode offers only few adaptable parameters, energy efficient optimizations for the nonbeacon mode are in general done by extending or modifying the 802.15.4 protocol stack. SCP [7], an example of a WSN-MAC protocol, runs on 802.15.4 compliant radios, but replaces the CSMA-CA channel access 
by a synchronized adaptive channel polling scheme. Ye et al. showed that this enables sensor node duty cycles below $0.1 \%$ and enables multi-hop streaming with only small end-to-end delay. Recently, we proposed a self-organizing sleep scheduling solution operating above the 802.15.4 MAC [8]. By loosely synchronizing with their neighbors, low duty cycled sensor nodes achieve good packet delivery ratios under a distributed routing scheme.

None of the above mentioned studies considers the early stages of the network life, but all works concentrate on the situation where the network is already fully operational. One of the first performance evaluations of 802.15 .4 by Zheng and Lee [9] is one of the rare works covering the initial network stage. Among many other aspects, the authors studied the association procedure for the case of beaconed networks and proposed an optimization. They used ns- 2 for their simulation studies and their code is still the base of the actual WPAN ns-2.33 simulation framework which we adapted for our purposes. Recently, Zhang et al. [10] showed how to speed up the association procedure in beaconed networks. For this purpose, the number of sent primitives and thereby the collision probability of command messages were reduced. This approach is only suitable for beaconed star topologies, as sending less primitives causes conflicts to occur in networks with several FFDs.

According to Zheng and Lee, an efficient association procedure is the basis for an efficient routing tree establishment 9. This idea was extended by Cuomo et al. 11] who exploit the parent-child relationship resulting from the association procedure for establishing a routing tree rooted in the PAN coordinator. As no additional overhead for initial route establishment is required, HERA, as this algorithm is called, outperforms AODV in simulations in terms of packet loss, delay and energy consumptions. The association procedure was not considered in this study, but of course heavily influences HERA's performance.

\section{The Nonbeacon 802.15.4 Association Procedure}

In this section, we review the key facts of the nonbeacon association mechanism as defined in [1], before we introduce our extensions for low-power WSNs.

\subsection{Basic Mechanism}

We consider 802.15.4 devices working in the unlicensed 2.45 GHz frequency band in the following. In this band, a starting PAN coordinator chooses one out of 16 available channels to operate the PAN it is going to coordinate. A node $x$ willing to associate to this PAN executes an active channel scan, i.e. it broadcasts a beacon request to all available channels. If the PAN coordinator or an already associated FFD receive such a request, they answer by sending a beacon. After having sent the beacon request, $x$ waits $\left[0.015 \cdot\left(2^{\text {ScanDuration }}+1\right)\right.$ sec for a response [1]. As no default value for $0 \leq$ ScanDuration $\leq 14$ is given, we adopt Zheng and Lee's proposal of ScanDuration $=4$, resulting in 0.26 sec per channel [9]. We further apply the low-power optimization proposed by [9] to scan 
only 3 channels. Together with processing times and state transitions, a node spends slightly more than $t_{\text {scan }}=0.78 \mathrm{sec}$ for a channel scan.

After the scan, $x$ sends an association request command message to one of the devices from which it received a beacon. 1 does not specify which out of several beacon senders to choose, we therefore follow again [9] and let $x$ send the association request to the quickest respondent $y$. After having received the association request, $y$ immediately sends back an acknowledgment, which $x$ answers by a data request command after aResponseWaitTime $=0.49 \mathrm{sec}$. Meanwhile, $y$ has to check, whether $x$ may associate. If yes, $y$ sends an acknowledgement and an association response command frame back to $x$ which in turn is acknowledged. Conditions for rejection are not mentioned in the standard [1], in our study, nodes are therefore always allowed to associate.

\subsection{Extension for Low-Power WSNs}

The 802.15.4 standard is optimized for star topologies, and proposes to realize larger deployments by using several PAN coordinators. Thus, the case where $x$ receives no beacon in response to its channel scan is not supposed to happen and thus not handled. For large low-power WSN deployments with only one PAN coordinator, it may in contrast occur quite frequently that a node receives no answer to its channel scan and fails to associate at first attempt. Possible reasons are that the PAN coordinator is out of reach, already associated FFDs are in sleep mode or packets are lost. Zheng and Lee [9] identified this problem and proposed that each node failing to associate should retry to associate $a=1$ sec later. They proved the effectiveness of this mechanism for large beacon-enabled topologies where nodes are always active.

Our studies showed, that if nodes are periodically active and inactive, this solution may cause sensor nodes to try and retry to associate during their neighbors inactivity phases. We investigate whether for this scenario a randomization of the association retry interval $a$ is beneficial and examine the impact of $a$ 's length on the association procedure performance. In addition, we propose two extensions for the node behavior: The greedy behavior causes a sensor node to immediately retry to associate after an unsuccessful channel scan. The altruistic behavior causes a successfully associated node to listen to the channel some time before starting its regular sleep-wake cycle. If the node receives any beacon requests, it starts to sleep as soon as it has completed all associations it participated in, otherwise the node starts to sleep after a period $t_{\text {alt }}$.

\section{Methodology}

Many factors influence the performance of a WSN and especially the association procedure in nonbeacon-enabled 802.15.4 WSNs. To investigate the performance of the association procedure under varying environmental conditions and design choices are abstracted by a set of factors which is discussed in Section 4.1. To evaluate the performance of the association process, we use the metrics introduced in Section 4.2. Details on the used energy model are given in Section 4.3. 


\subsection{Considered Factors}

We aim at improving the performance of a given 802.15.4 WSN deployment by adapting the association procedure. We abstract this problem by introducing a set of hard factors describing the application requirements and environmental conditions which can not or only hardly be changed. The degree of freedoms for tuning the association procedure are represented by a set of soft factors.

\section{Hard Factors}

- Node deployment (dep): Describes the strategy by which the positions of the sensor nodes are chosen.

- Average node degree (d): The number of other nodes each sensor node may on average communicate with is determined by the transceiver transmission output power and the density of the node deployment.

- Coefficient of variation of node activation time (c): Each sensor node is activated a randomly distributed time $t_{0}$ after the PAN coordinator has been switched on. Different values of $c$ model different starting behaviors.

- Network clock (T): Determines the responsiveness of the WSN.

- Activity factor $(\alpha)$ : An energy efficient operation is abstracted by assuming that all sensor nodes are active $\alpha T$ and sleep for $(1-\alpha) T$, where $0<\alpha \leq 1$.

- Initial tolerance time $(\Delta)$ : Determines when the WSN has to be functional.

\section{Soft Factors}

- Association retry interval (a)

- Randomization of a (rand)

- Greedy association (gr)

- Altruistic association (alt)

\subsection{Performance Metrics}

For their study of the association process, Zheng and Lee proposed two metrics: the successful association rate giving the percentage of devices which succeeded to associate and the association efficiency, indicating how much attempts the devices had to make for associating [9]. We extend this framework and use the following three network level metrics to evaluate the association process performance for a certain factor value combination:

Association Success. $s_{A}=1$ if all nodes of the PAN could associate during $\Delta$ and 0 otherwise.

Association Time. $t_{A}$ is the time until the last node of the PAN has associated. If not all nodes could associate, $t_{A}=\Delta$.

Association Power Consumptions. $E_{A}$ denotes the energy consumptions of the node of the PAN which required the largest amount of energy for associating. $E_{A}$ does not include the energy consumptions required for sending out association responses, but focuses on the nodes trying to associate. 


\subsection{Energy Model}

An exact calculation of $E_{A}$ has to include characteristics on all mote subsystems. As our study focuses on reducing the communication related energy consumptions, we aim only at estimating the energy consumptions of the transceiver. For this purpose, we use the state machine model proposed by Wang and Yang [12], who extracted values for current consumptions and transition times from transceiver data sheets and assumed a typical voltage of $U=1.8 \mathrm{~V}$. Experiments with the 802.15.4-compliant CC2420 [13] showed that this approach together with an exact model of the communication behavior closely estimates the current consumptions. Transition power consumptions are calculated as the average of the initial and final state power consumptions [12.

One component of the energy node $x$ requires for associating, $E_{A}(x)$, is $E_{\text {start }}$ which is consumed when the node initially activates its transceiver, i.e. for the transition from "Power Off" to "Receive" (RX) state. Next, $E_{\text {scan }}$ is consumed for each of the $n \geq 1$ channel scans. If the association procedure is not greedy, during each of the $n-1$ periods where $x$ waits for retrying to associate, $E_{R}$ is consumed. $E_{R}$ is obtained by adding the energy consumptions for transitions from and to "Power Save" state to the energy consumptions in "Power Save" for the remaining time of $a$. For the association command message exchange, $E_{a}$ and the optional altruistic phase, $E_{a l t}$ are required. $E_{\text {scan }}, E_{a}$ and $E_{a l t}$ are estimated by multiplying the energy current consumptions in RX state by the time required for these actions and the voltage $U$. As CC2420 consumes slightly more energy for receiving than for transmitting at the highest output power 13, (which we assume to be the case in our study), this estimation is close to the real consumptions and sufficient for the purposes of this study.

\section{Simulation Results}

To examine the effect of our association procedure extensions, we use the ns-2.33 802.15.4 stack and extend the association mechanism as discussed in Section 3.2 The impact of all factors besides the node deployment is examined using a $2^{k}$ factorial design study in Section 5.1. The obtained results allow to identify beneficial influence of the greedy and altruistic mechanism which we analyze more closely for different topologies in Section 5.2 .

\subsection{A $2^{7}$ Factorial Design Study}

Our simulations confirmed the evident fact that the topology has the strongest influence on the performance of the association procedure. To examine the other factors' influences, we consider a simple topology where 9 sensor nodes and one PAN coordinator are arranged on a line, with the PAN coordinator at the left edge. $\Delta=5 \mathrm{~min}$ and $T=1 \mathrm{sec}$ are used for this experiment, but the results are similar for other parameter choices. For each of the 7 remaining free factors, a high (+) and low (-) level as summarized in Table 1 are chosen. 
Table 1. Considered experimental factors and their levels

\begin{tabular}{|l||c|c|c|c|c|c|c|}
\hline Factor & $d$ [nodes] & $c$ & $\alpha[\%]$ & alt & gr & $a$ & rand \\
\hline \hline Level $(-)$ & 1.8 & 0 & 1 & no & no & $0.1 T$ & no \\
\hline Level $(+)$ & 6 & 1 & 25 & yes & yes & $5.1 T$ & yes \\
\hline
\end{tabular}

We model the node transceivers to correspond CC2420 transmitting at the highest $(0 \mathrm{dBm})$ possible output power. The radio propagation is abstracted as a disc model, and the two-ray ground propagation model proposed by [9] is used. Together with CC2420's typical receiver sensitivity of $-95 \mathrm{dBm}$ [13] this causes each transmission within $20 \mathrm{~m}$ to succeed. We consider inter-node spacings of 20 and $5 \mathrm{~m}$, each node can thus communicate with 1 or 4 neighbors per direction. Averaged over 10 nodes, this results thus in a low value of $d=1.8$ and a high value of $d=6$. Startup times distributed with $c=0$ stands for all nodes starting at exactly the same time, $c=1$ corresponds to an exponential distribution with the same mean. $\alpha$ reflects a good lower and an absolute upper bound for a lowpower network duty cycle. Low and high values for the factors rand, gr and alt correspond to using these features or not. If the altruistic mechanism is used, we parameterize $t_{\text {alt }}$ to be slightly longer than the time required for a channel scan, $t_{\text {alt }}=t_{\text {scan }} \cdot 1.1=0.86$ sec. $a$ is chosen in dependence of the system clock and varies between a very small and a very large value. To randomize $a$, we multiply it by a random variable $X$ which is uniformly distributed in the interval $(0,1]$. The effects of using other distributions for $X$ have not been considered in this study. Other choices for the levels are imaginable, but our experiments showed that using other level values just slightly changes the results.

To get an overview on the factors' influence, we examine the performance of all $2^{7}$ factor combinations or design points. For each design point, the performance of the association mechanism is evaluated by the following simulation run: at time 0, the PAN coordinator is activated and begins starting a PAN. The PAN coordinator is always active during the simulation run [1. Each of the sensor nodes is activated at a randomly distributed time $t_{0}$ with mean 30 seconds and coefficient of variation as specified by $c$. After the activation, each node tries to associate as described in Section 3.1 using the extensions introduced in Section 3.2 with the parameters set for this design point. After having successfully associated and after an optional altruistic period, the node starts to sleep regularly for $(1-\alpha) T$. At the end of each simulation run, the system responses, i.e. the metrics $s_{A}, t_{A}$ and $E_{A}$ defined in Section 4.2 are collected. To obtain statistically significant results, the responses for each design point are averaged over 100 simulation runs.

Main Effects. The influence of factor $x$ on the system performance in terms of metric $y$ is characterized by its main effect, $e_{x}(y)$. It is obtained as the average change in $y$ due to moving $x$ from (-) to $(+)$ while keeping all other factors fixed:

$$
e_{x}(y)=\frac{\bar{y}_{x^{+}}-\bar{y}_{x^{-}}}{2}
$$




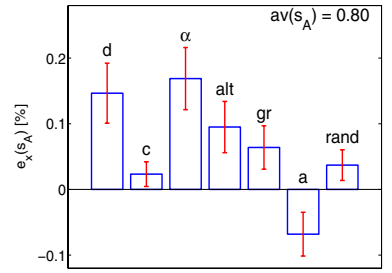

(a) $s_{A}$

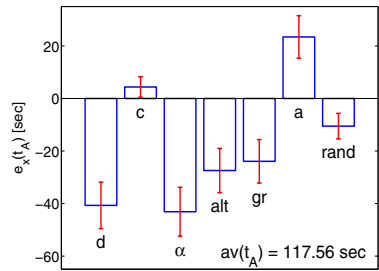

(b) $t_{A}$

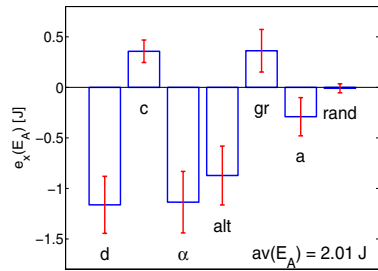

(c) $E_{A}$

Fig. 1. Main effects of the considered factors on the different metrics

where $\bar{y}_{x^{+}}$and $\bar{y}_{x^{-}}$denote $y$ averaged over all design points where $\mathrm{x}$ is at its high level and low level respectively [14.

In Fig. 1] we visualize the main effects $e_{x}$ of the 7 different considered factors. From left to right, the subfigures contain representations for the association success, the association time and the association energy consumptions of the network. Together with the main effects, their 95\%-confidence intervals are shown to verify, that only some of the effects are not statistically significant, as their confidence intervals include 0 . Additionally, we show the corresponding system response averaged over all $2^{7}$ design points in each figure.

Let's discuss the average system responses first. The association success $s_{A}$ is the probability that all nodes associate within $\Delta$. As $s_{A}=0$, if all but one node were able to associate, $s_{A}$ averaged over all design points and 100 runs is only 0.8. The probability that an individual node associates, is slightly higher and averages to 0.88 . Obviously both probabilities converge to 1 for larger values of $\Delta$. Note that the association energy consumptions for this topology averaged over all design points and runs are equal to $2 \mathrm{~J}$. This corresponds to less than $0.01 \%$ of the theoretical capacity of two AA batteries [15] and seems to be neglectable when compared to the energy consumptions during the WSN lifetime. However, already in this simple topology the minimal and maximal $E_{A}$ of $0.2 \mathrm{~J}$ and $10.4 \mathrm{~J}$ differ strongly, the inherent energy saving potential of the association phase must thus not be underestimated.

The main effects of the factors shown in Fig. 1 illustrate that some factors have a stronger influence than others and that the factor impact differs between the metrics. A closer look reveals, that the hard parameters $d$ and $\alpha$ have a larger influence then all other parameters: the association procedure will succeed with high probability, rather quick and at low energy costs in dense and high duty cycled topologies. While this is quite evident, the influence of a randomized startup point characterized by $c$ 's main effect is ambiguous. $c>0$ is suitable for increasing the association success as it decreases the probability of packet collisions especially in dense networks, but results in slightly increased time and energy consumptions.

As the hard parameters can in general not be influenced, the analysis of the soft parameters is more profitable for optimization purposes. The randomization 
of the association retry interval and to a stronger degree the altruistic mechanism are the only soft parameters which have a positive influence on all three considered metrics. The energy saving potential of the altruistic mechanism is quite large as the energy savings of nodes which associate earlier outweigh the energy consumptions of nodes altruistically delaying their transition to power save mode. The greedy mechanism and a small association retry interval increase the association success and the association speed but lead to increased energy consumptions by triggering too many unnecessary channel scans.

Interactions. Considering just the main effects of the factors for deciding the parametrization of the association mechanism would lead to wrong decisions, if interactions between factors are existing, i.e. if the influence of one factor varies in dependence of the behavior of other factors. To examine this, we consider the two-way interaction effect of factors $x$ and $z$ on systems response $y, e_{x y}(y)$, which is computed as the average difference of $x$ 's effect when $z$ is at its $(+)$ and the effect of $x$ when $z$ is at its (-) level [14]:

$$
e_{x z}(y)=\frac{1}{2}\left(\left(\bar{y}_{x^{+} z^{+}}-\bar{y}_{x^{-} z^{+}}\right)-\left(\bar{y}_{x^{+z^{-}}}-\bar{y}_{x^{-} z^{-}}\right)\right),
$$

where analogous to Eq. (1), $\bar{y}_{x+z}+$ denotes $y$ averaged over all design points where both $x$ and $z$ are at their $(+)$ levels.

The interactions of all considered responses are similar, we therefore only illustrate the interactions on the energy consumptions in Fig. 2. In the subfigure which is at row $z$ and column $x$ of the figure matrix, a solid line is drawn between the average system response when $x$ is at its $(-)$ and $(+)$ level, while $z$ is at is (-) level, $\bar{y}_{x^{-} z^{-}}$and $\bar{y}_{x^{+} z^{-}}$, while a dashed line connects the average system response when $x$ is at its $(-)$ and $(+)$ level, while $z$ is at is $(+)$ level, $\bar{y}_{x^{-} z^{+}}$and $\bar{y}_{x^{+} z^{+}}$.

Non-parallel lines represent interactions between two factors, it gets thus quickly clear, that nearly all factors are interacting. Fig. 2]illustrates that factors with strong main effects also have strong interactions and that factor levels have to be adapted under the consideration of other factors levels. Often a factor's effect is amplified or reduced by another factor's level, as it is e.g. the case for all soft parameters which have a less stronger effect, if the activity is high and the network is dense. All in all, the analysis of interactions demonstrates, that any deeper analysis of the association mechanism has to be done considering different network configurations and that sparse and low-duty cycled network designs need to be optimized more carefully.

\subsection{Combining the Greedy and the Altruistic Mechanism}

In the last section we demonstrated, that out of the soft factors, the greedy and the altruistic mechanisms have the strongest effects on the association success and speed. We saw also, that the altruistic mechanism reduces the increased energy consumptions of the greedy mechanism (c.f Fig. 2). In this section we verify if this holds also for larger topologies and investigate to what degree the association procedure in a given sparse low-power WSN deployment can be improved using both mechanisms. 


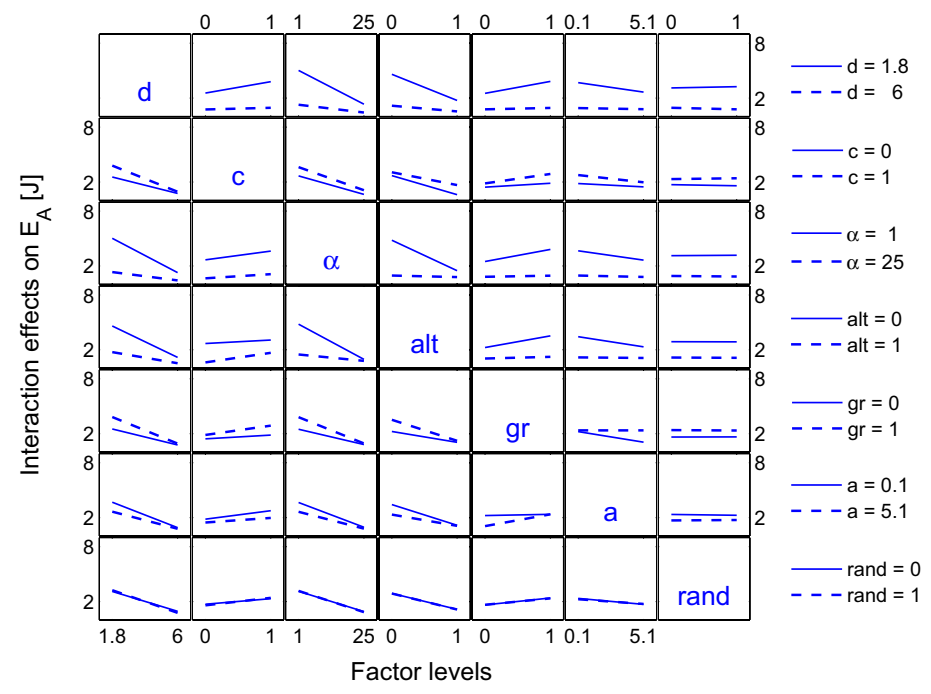

Fig. 2. Interaction effects on the association energy consumptions

We consider an exemplary monitoring application, where an area of $60 \times 60 \mathrm{~m}$ is covered with 24 sensor nodes and one sink node acting as PAN coordinator. Small energy consumptions together with acceptable responsiveness are abstracted by $\alpha=1 \%$ and $T=1$ sec. To examine the influence of the node deployment and the sink position, we use randomly generated topologies: A connected graph with 25 nodes results either from chosing positions on a grid with $12 \mathrm{~m}$ in between or uniformly distributing nodes over the square to cover. In both cases, one random position is chosen to be the position of the sink. Averaging the mean node degree over 100 different random and grid topologies results in $d=6$ and $d=3.2$ respectively. To analyze the benefits of the proposed mechanisms, we do not randomize the associatione retry interval and set $a=0.5 T$. Different possibilities for activating the sensor nodes are abstracted by choosing $t_{0}$ as randomly distributed with coefficient of variation $c \in\left\{0,1 e^{-3}, 1 e^{-2}, 1 e^{-1}, 0.577,1,2\right\}$ and mean $30 \mathrm{sec}$. For a closer examination of the altruistic mechanism, values for $t_{\text {alt }}=\left\{0, t_{\text {scan }}, t_{\text {scan }}+T, t_{\text {scan }}+2 T, t_{\text {scan }}+5 T, t_{\text {scan }}+10 T\right\}$ are used.

In Fig. 3 we depict the association success and energy consumptions resulting from $\Delta=5 \mathrm{~min}$ and a strongly varying node activation time, i.e. $c=2$. Results averaged over 100 runs with their 95\% confidence intervals are shown which are quite large due to the high variance of $t_{0}$. Results obtained with the greedy mechanism are shown by shaded bars. The six bars of each of the four groups depict the considered values of $t_{\text {alt }}$ in increasing order. Furthermore, we show results for the random (the two left bar groups in each subfigure) and the grid topologies (the two right bar groups). This illustrates, that due to the smaller average degree most effects are stronger in the grid than in the random topologies and demonstrates again that sparser topologies need more optimization effort. 


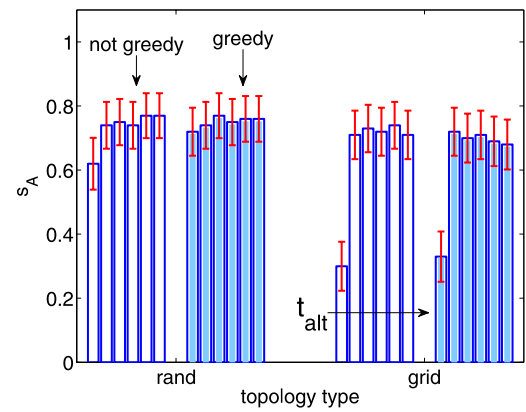

(a) $s_{A}$

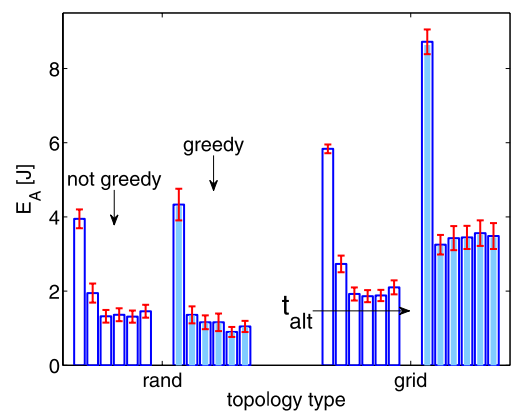

(b) $E_{A}$

Fig. 3. Effects of the altruistic and greedy mechanism for a high startup variation

As the node activation time is varying very strongly, many nodes are starting close to $\Delta$, causing the association success to be small, especially in the sparser grid topologies. Both mechanisms are able to increase the association success and decrease the association energy consumptions but to a different degree: The clear difference between the size of the first bar in each group $\left(t_{\text {alt }}=0\right)$ and all other bars demonstrates, that the altruistic mechanisms has a stronger positive influence on both metrics than the greedy mechanism (non shaded vs. shaded bars). Combining the greedy and the altruistic mechanisms positively influence the system performance, as the energy penalties resulting from the use of the greedy mechanism are alleviated while the time and energy reductions of both mechanisms also result from their combined use.

According to the results illustrated in Fig. 3, the exact parametrization of $t_{\text {alt }}$ is affecting the system performance differently if the greedy mechanism is used. The association success is directly increasing with $t_{\text {alt }}$ we thus analyze the more interesting trade-off between association speed and energy consumptions in dependence of the startup variation. For this purpose, $\Delta$ is set to 10 minutes which allows all nodes in the considered topologies to associate in nearly all cases. Results from this experiment for both topology types were similar, but stronger for the

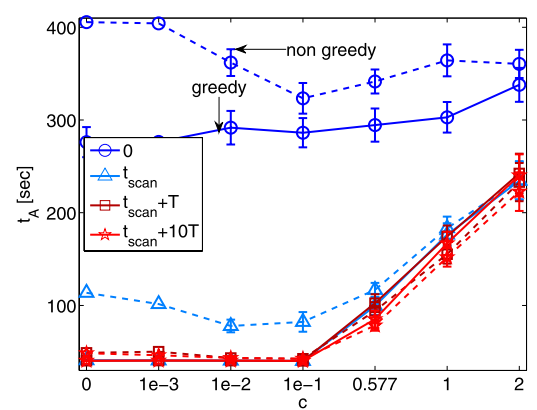

(a) $t_{A}$

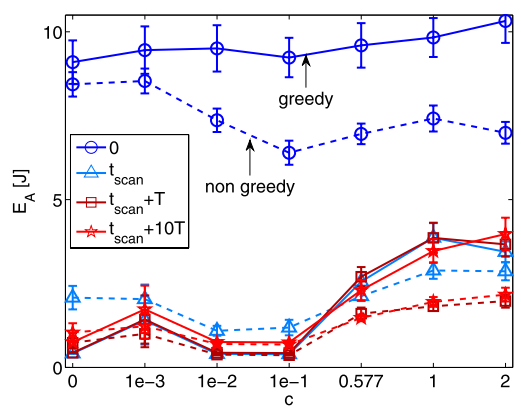

(b) $E_{A}$

Fig. 4. Altruistic and greedy mechanism under varying starting time variations 
grid topologies. The association time and energy consumptions averaged over 100 simulation runs together with their $95 \%$ confidence intervals are plotted against $c$ in Fig. 4. Different values of $t_{\text {alt }}$ are distinguished by different markers. The greedy and the non greedy behavior are shown with solid and dashed lines respectively.

At first glance it gets clear, that $t_{\text {alt }}=0$, i.e. a non altruistic behavior, leads to the slowest association and highest energy consumptions. The altruistic mechanism is improving the performance more than the greedy mechanism. The benefits of the latter are moreover strongly depending on the startup variation: While a greedy behavior together with a small $t_{\text {alt }}>0$ increases the association speed and decreases the association energy consumptions in networks with small startup variation, this effect is inversed for larger values of $c$. This demonstrates, that for the exact parametrization of $t_{\text {alt }}$ a balanced solution between association speed and energy consumptions has to be found: the association speed always increases with $t_{\text {alt }}$, but if $t_{\text {alt }}>t_{\text {scan }}$ is chosen too large, too much energy is wasted by unnecessarily listening to the channel. All in all, Fig. 4 demonstrates that in the scenario we investigated, adapting the usage of the greedy and altruistic mechanism to the WSN deployment enables accelerations over $300 \mathrm{sec}$ and energy savings over 5 J. Comparable numbers will result from topologies of the same size and will be larger for larger deployments.

\section{Conclusion and Outlook}

In this paper we examined possibilities for optimizing the association process in a low-power 802.15.4 nonbeacon sensor network. For this purpose, we proposed four enhancements for the procedure as described in the standard and carried out an extensive simulation study to identify the effects of our improvements. Our results showed, that the influence of factors given by the application requirements is always larger than the improvements achieved by tuning the association mechanism. However, significant performance gains in terms of association probability, speed and energy consumptions may be achieved, if the additional mechanisms we proposed are well parameterized. The altruistic and the greedy behavior are especially promising, as they allow to increase the performance of the association procedure by adapting it to the network characteristics.

All in all, our results illustrate the inherent optimization potentials of the often neglected 802.15.4 association procedure. A successful starting phase is the foundation of each successful WSN deployment and especially of 802.15.4 WSNs, our future works will therefore be dedicated to further optimizing the association procedure. As the startup behavior of the nodes is strongly influencing the network performance, we will also study optimization possibilities for the node activation strategy.

\section{Acknowledgements}

The authors would like to thank Phuoc Tran-Gia and Dirk Staehle for valuable comments and fruitful discussions. Special thanks go to Sebastian Deschner for his help during the simulation process. 


\section{References}

1. IEEE Computer Society: IEEE Standard for Information technology - Part 15.4: Wireless MAC and PHY Specifications for WPANs (September 2006)

2. ZigBee Alliance: ZigBee Specification (January 2008)

3. HART Communication Foundation: WirelessHART Technical Data Sheet (May 2007)

4. Kohvakka, M., Kuorilehto, M., Hännikäinen, M., Hämälinen, T.D.: Performance analysis of IEEE 802.15.4 and ZigBee for large-scale wireless sensor network applications. In: PE-WASUN 2006 (2006)

5. Latré, B., Mil, P.D., Moerman, I., Dhoedt, B., Demeester, P.: Throughput and Delay Analysis of Unslotted IEEE 802.15.4. Journal of Networks 1(1) (May 2006)

6. Kim, T.O., Kim, H., Lee, J., Park, J.S., Choi, B.D.: Performance Analysis of IEEE 802.15.4 with Non-beacon-enabled CSMA/CA in Non-saturated Condition. In: Sha, E., Han, S.-K., Xu, C.-Z., Kim, M.-H., Yang, L.T., Xiao, B. (eds.) EUC 2006. LNCS, vol. 4096, pp. 884-893. Springer, Heidelberg (2006)

7. Ye, W., Silva, F., Heidemann, J.: Ultra-Low Duty Cycle MAC with Scheduled Channel Polling. In: SenSys 2006 (2006)

8. Staehle, B., Hoßfeld, T., Vicari, N., Kuhnert, M.: A Cross-Layer Approach for Enabling Low Duty Cycled ZigBee Mesh Sensor Networks. In: International Symposium on Wireless Pervasive Computing 2008, Santorini, Greece (May 2008)

9. Zheng, J., Lee, M.J.: A Comprehensive Performance Study of IEEE 802.15.4. In: Sensor Network Operations, pp. 218-237. IEEE Press, Los Alamitos (2004)

10. Zhang, F., Wang, F., Dai, B., Li, Y.: Performance Evaluation of IEEE 802.15.4 Beacon-Enabled Association Process. In: AINAW 2008 (2008)

11. Cuomo, F., Luna, S.D., Monaco, U., Melodia, T.: Routing in ZigBee: Benefits from Exploiting the IEEE 802.15.4 Association Tree. In: IEEE ICC 2007 (2007)

12. Wang, Q., Yang, W.: Energy Consumption Model for Power Management in Wireless Sensor Networks. In: SECON 2007 (2007)

13. Texas Instruments: 2.4 GHz IEEE 802.15.4 / ZigBee-ready RF Transceiver. Texas Instruments (2006)

14. Montgomery, D.C.: Design and Analysis of Experiments, 6th edn. John Wiley and Sons, Chichester (2005)

15. Energizer: Alkaline Technical Information (2008), http://data.energizer.com 